\title{
A Fuzzy Connectivity Tree for Hierarchical Extraction of Venous Structures
}

\author{
Silvana Dellepiane, Lorena Novelli, Michele Bruzzo, and Marco Antonelli \\ University of Genoa \\ DIBE - Department of Biophysical and Electronic Engineering \\ Via Opera Pia, 11A - 16145 Genova, ITALY \\ Ph. +39-010-3532754, Fax +39-010-3532134 \\ silvana@dibe.unige.it
}

\begin{abstract}
In Magnetic Resonance Angiography (MRA), blood vessels show very high grey levels resulting from the use of a contrast agent. This leads to develop a formula, named $\beta$-connectedness, derived from fuzzy-connectedness theory [1],[2]. $\beta$-connectedness is described here as a specific case of more general $\chi$-connectedness [3], for it allows one to improve the tracking of bright structures and, consequently, vessel extraction. In the computation of fuzzy connectedness, a widely used approach is based on an adaptive growing mechanism that follows the best paths starting from a reference seed point. As a consequence, a hierarchical tree is generated. Contrary to the aforesaid approach, here we propose to exploit such a tree information and we define the connectivity level as an additional parameter useful in the analysis of image data. The method presented in the paper deals with a new kind of connectivity that depends on the position of each pixel in the growing tree. The detection of fine structures is thus improved, as demonstrated by preliminary results on 3D MRA volumes.
\end{abstract}

\section{Introduction}

Segmentation of venous structures is very important to study different diseases of vascular trees and for surgical planning. The MRA (Magnetic Resonance Angiography) acquisition process is able to detect tissues and a contrast agent inside a body, then the blood flow can be observed. In addition, MRA acquisition suffers from common artifacts, like the partial-volume effect.

Classical methods that try to use pre- and post- processing techniques in order to enhance segmentation results exhibit the major drawback of deleting thin structures that are necessary to make a correct diagnosis.

The present paper starts from the fuzzy techniques presented in [2], [3] and proposes two main improvements in order to obtain good results even in the case of thin, inhomogeneous structures. The innovative application of fuzzy connectivity turns out to be very effective in describing region topology. 
As blood vessels show very high grey levels in MRA for the presence of a contrast agent, here a new computation of fuzzy connectedness [2] is proposed, that is more sensitive to bright structures. In the following, it is named ' $\beta$-connectedness'.

In addition, the order of analysis inherent in fuzzy-connectedness is adopted to take into account the relationship between a reference point (the seed point) and the other voxels of the image dataset. Toward this end, the hierarchical tree derived from the adaptive scanning mechanism described in [2] is exploited to understand the growth process and becomes an additional feature for image analysis. This idea allows us to develop a new definition for the degree of connectivity, namely, the connectivity level, which depends on the position of each voxel in the tree.

\section{Related Works}

As regards the application of fuzzy theory to medical image segmentation, two main approaches are available. The first (i.e., the one based on $\chi$-connectedness, as described in [2] and [3]) represents the basis for the proposed method, whereas the second was originally proposed in [4] and is based on the ' $\kappa$-connectedness'. Both fuzzy-connectedness approaches integrate pixel similarity with topological connectivity.

Concerning the artery/vein separation problem, a larger number of features have to be used together with an a-priori model of anatomical properties and shapes. In this context, starting from $\kappa$-connectedness, a novel approach is presented in [5]. In addition to intensity-affinity and adjacency concepts, scale-based affinity is considered to take into account object dimensions.

In another paper [6], the Ordered Region Growing (ORG) algorithm is proposed to extract vessel the axis of a vessel by specifying the vessel endpoints with respect to a reference point. By acting as a sort of region-growing segmentation process, this algorithm, keeps track of the growing direction, and represents a vascular structure as a graph where connectivity among all voxels is considered. This technique de facto applies the growing mechanism proposed in [2], as it selects, starting from the seed voxel, neighbouring elements with the maximum grey level. The graph stores the relationships between a seed point and its descendants, and is used to extract the skeleton of the veins.

The main advantage is a low computational burden, whereas the main drawbacks result from an inaccurate connectivity estimation, which is more evident in the case of dark regions where vessels show a low contrast and in the case of large vessels. Finally, no segmentation result is achieved.

In conclusion, the idea of exploiting the order of analysis derived from the actual growing process has been very seldom proposed in the literature, and is presented here for the first time in conjunction with segmentation. 


\section{Fuzzy-Connectedness Theory}

As can be seen in the literature, the use of fuzzy theory, in particular, the application of fuzzy connectedness [1] allows one to develop different formulae ([2],[4]) suitable for the extraction of inhomogeneous structures, as in the cases of medical and remotesensing applications. In this paper, a specific type of medical images, i.e. those of Magnetic Resonance Angiography (MRA), is used.

For the sake of clarity, some formulae (taken from [2]) concerning the modified field $\chi_{a}$ and the relative $\chi$-connectedness are recalled.

If we denote by $a$ the seed point that belongs to the structure we want to extract, we can define a modified field $X^{a}$ where the seed point has the maximum value and each point of the field can be obtained as follows:

$$
X^{a}(p)=1-|\eta(p)-\eta(a)|
$$

where $\eta(p)$ is the fuzzy field derived from the original image.

Equation 1, provides the grey level mapping, which is shown in Fig. 1: one can notice the maximum membership of the seed point.

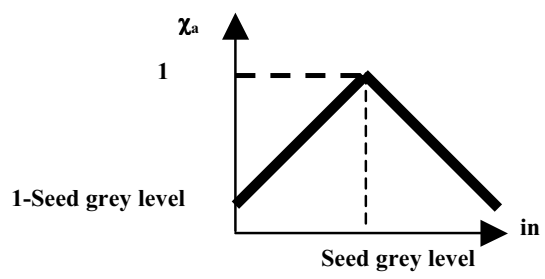

Fig. 1. The modified value $\chi_{\mathrm{a}}$ as a function of the original value (in).

The connectivity associated with the seed point $a$ is found by applying the classical formula of fuzzy connectedness [2] to $X^{a}$ :

$$
C_{X^{a}}=c_{X^{a}}(p)=\operatorname{conn}\left(X^{a}, a, p\right)=\max _{P(a, p)}\left[\min _{z \in P(a, p)} X^{a}(z)\right]
$$

Equation 2 defines " $\chi$-connectivity" or "intensity-connectedness" [2]. It allows one to create a connectivity map where each image element has a grey level dependent on the degree of connectivity with respect to the seed point $a$. After the computation of this map, it is possible to extract the object of interest by performing a thresholding operation. The application of Equations 1 and 2 and the thresholding phase allow one to carry out the algorithm named Isocontour. 


\subsection{Application of Fuzzy Isocontour to Bright Structures}

Contrast-Enhanced Magnetic-Resonance-Angiography (CE-MRA) is characterized by high intensity levels corresponding to blood vessels and resulting from the injected contrast agent. Since the fuzzy-Isocontour approach makes use of an image transform $\left(X^{a}\right)$ designed to deal with the most general cases, here we propose a new transform, named $\beta_{a}$, that is aimed at the segmentation of bright structures, like blood vessels in CE-MRA images.

$\beta_{a}$ is a variant of the $X^{a}$ formula (1) and privileges the voxels with larger intensity values than the seed point. In other words, the seed point and the voxels with larger intensity values are characterized by the maximum membership and are therefore mapped with the maximum grey level. In this way, the brighter points are enhanced. The $\beta_{a}$ transform is expressed by the following formula:

$$
\beta_{a}=\left\{\begin{array}{cll}
1+\eta(p)-\eta(a) & \text { if } & \eta(p)<\eta(a) \\
1 & \text { if } & \eta(p) \geq \eta(a)
\end{array}\right.
$$

and is shown in Fig. 2.

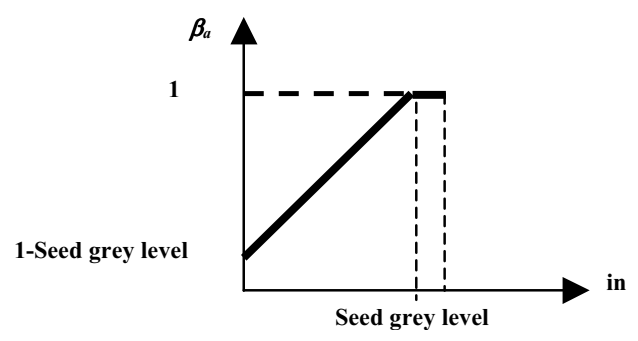

Fig. 2. The modified value $\beta_{a \text {. }}$. All the points with larger intensity values than the seed point are mapped with the maximum intensity value.

\section{A Fuzzy Hierarchical Tree}

The classical Isocontour algorithm, improved by the $\beta_{a}$ field, performs the segmentation process through a thresholding operation on the connectivity map.

In this section, a new segmentation algorithm exploiting the $\beta_{a}$-connectivity computation is presented that is able to extract a hierarchical tree providing for each pixel its connectivity value and its relationships with the other pixels. The idea is to organize into a dynamic structure the history of the connectivity processing: the root of the tree is represented by the seed point, and new nodes are added every time a new pixel is computed. It follows that, for each image element $i$, it is possible to know its connectivity value, the level of labelling $L_{i}$, and its relationships with the other elements.

The construction of the tree is based on the concepts of a generator pixel and a candidate pixel, as defined in [2]. Starting from the seed point, the voxels in the 
neighbourhood are considered. Such voxels are the seed point's sons, represent the first level of candidates, and are labelled according to Equation 2.

Among the level-1 candidates, the voxels with the maximum degree of connectivity are chosen; they become the level-1 generators, that is, the voxels that propagate the growing tree. In the next step, the neighbourhood of the level-1 generators are analysed: these voxels represent the level-2 candidates and the sons of the level-1 generators. In other words, the level-1 generators are the fathers of the level-2 candidates.

Each tree level is represented by voxels labelled in the same processing step, so the voxels that are at the $i$-th tree level are called i-level candidates, whereas the generators at the $i-1$ level are the fathers of the $i$-level candidates (Fig. 3).

It is obvious that each point $p$ is processed on the basis of a specific, content-based ordered image sequence that can be recovered by analyzing the tree levels. It is then possible to introduce a new parameter: the membership level $L$. Its value depends directly on the geometric distance of $p$ from the seed point, $d(p$, seed $)$, and on the connectivity value of $p$ with respect to the seed point, $\beta_{\mathrm{a}}(p)$. This allows one to regard the membership level as a connectivity measure, whose main properties are:

$L$ decreases with respect to $\beta_{\mathrm{a}}(p)$ and $\mathrm{d}(p$, seed $) \forall p \in I$;

$L \geq 0 \forall p \in I$, where $I$ is the image under analysis.

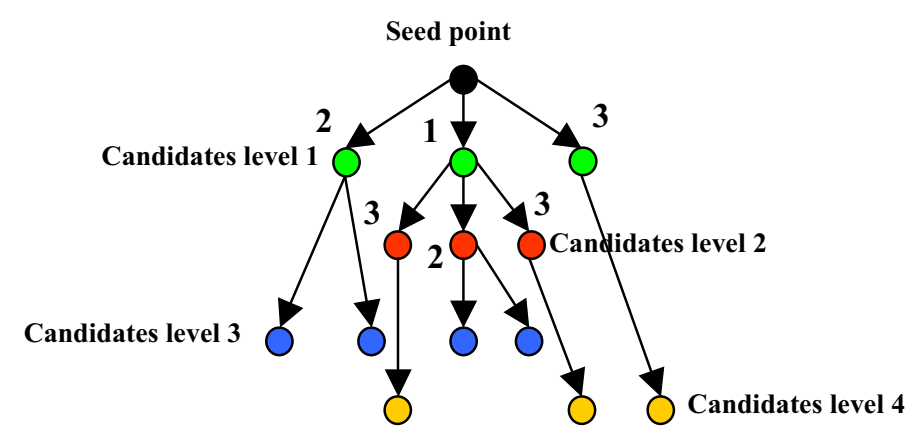

Fig. 3. An example of hierarchical tree. The nodes of the same colour represent the candidates at a specific processing level. The node numbers denote the generator levels.

To analyze the growing tree starting from the seed point, it is important to take into account the relations between the candidates and the seed point and those between the generators and the seed point. By studying these elements, it is possible to know the direction of growth of the structure to segment.

The analysis of the generators leads to the development of a tool, named 'Player', that allows a real-time visualization of the voxels with the temporarily largest membership values. This segmentation tool exploits the tree generated during the connectivity computation. The main target is to highlight only the level- $i$ generators with $i<M$, where $M$ is a value chosen by the user on the basis of the segmentation result displayed at run time. Figures 4 and 5 show examples where the generators are visualized during the first processing steps. As pointed out in the examples, a $k$ level generator is not necessarily a $k$-level candidate. 

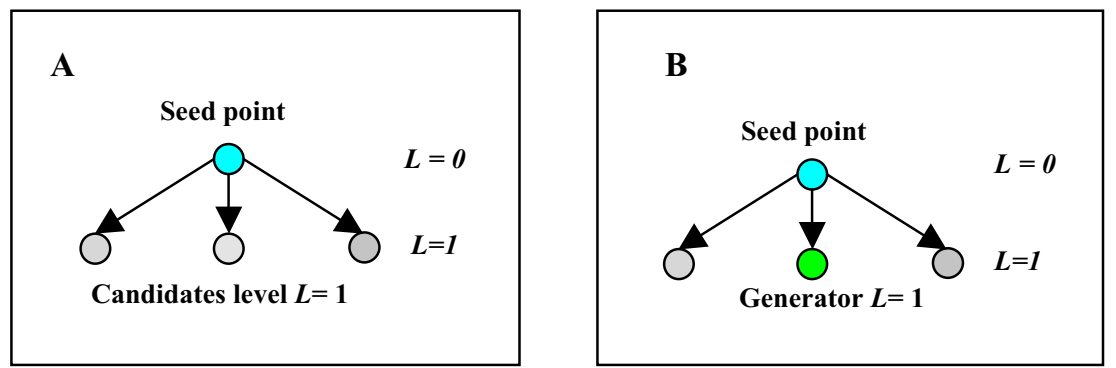

Fig. 4. Selected seed point and level-1candidates (A). Among all the level-1 candidates, the points with higher membership values with respect to the seed point, are considered generators; in this case, only one pixel has the maximum connectivity value (B)
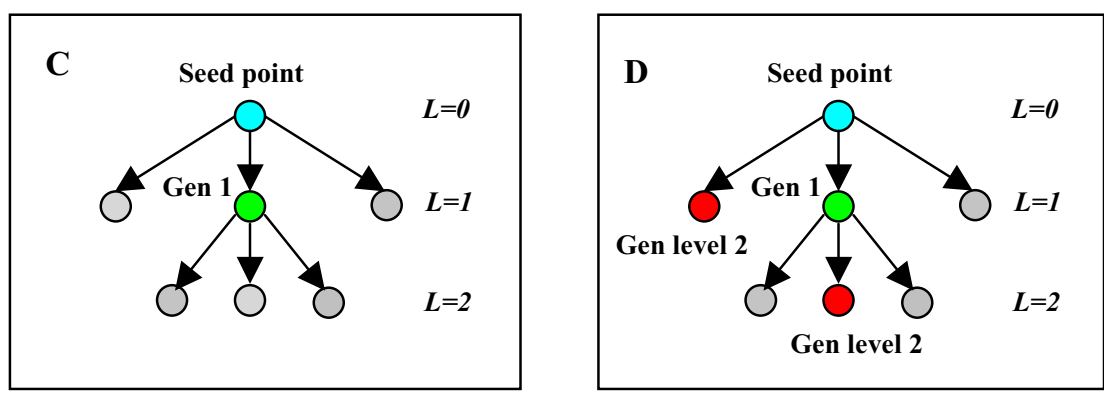

Fig. 5. Points labelled at level $L=2$ are the sons of level-1 generators (C). Visualization of level-2 generators (D). As can be seen, the generators in the same step, indicated by the red colour, belong to different levels $L$

The interaction mechanism is driven by the user. By clicking on a button, it establishes when and where the segmentation must stop, that is, when the whole object of interest is segmented. In the next example, the processing has been stopped at level 3 (Fig. 6).

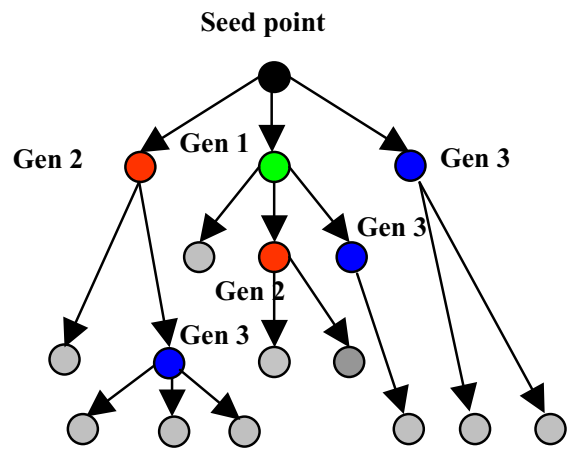

Fig. 6. The first three segmentation steps with the Player. The three colours represent different growing steps 


\section{Results}

The analysis of the fields $\mathrm{X}$ and $\mathrm{B}$ shows the better visualization of venous structures obtained by B. The $\beta_{\mathrm{a}}$ formula allows a whole blood vessel to be characterized by a very high grey level, and this makes the successive segmentation phase easier.

Figure 7A shows an MRA image acquired on the coronal plane. By positioning the seed point on the lateral branch, the $\chi_{a}$ and $\beta_{a}$ fields are obtained as displayed in Fig.s. $7 \mathrm{~B}$ and $7 \mathrm{C}$.

As shown in Fig. 8, the use of the $\chi_{\mathrm{a}}$ formula determines the extraction of a blood vessel, though with discontinuities. On the contrary, by using the $\beta_{\mathrm{a}}$ formula, the vessel is extracted without discontinuity regions.
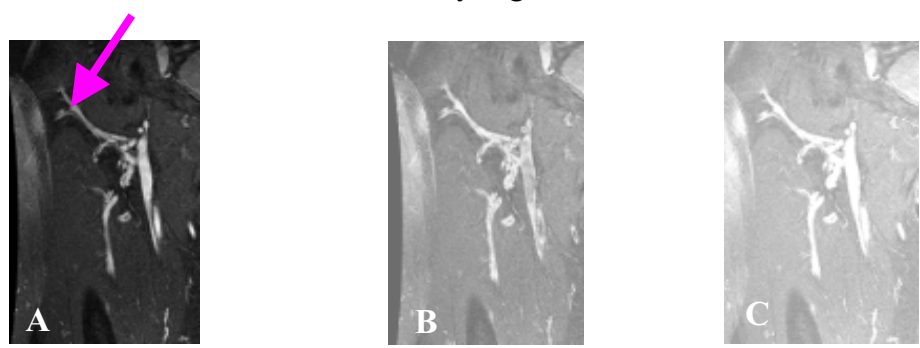

Fig. 7. Original coronal MRA image and selected seed point (A). Figure $\mathbf{B}$ shows the result of the $\chi_{a}$ formula calculation. As can be noticed (Figure $\mathbf{C}$ ), the $\beta_{a}$ formula provides a more homogeneous representation of the blood vessel
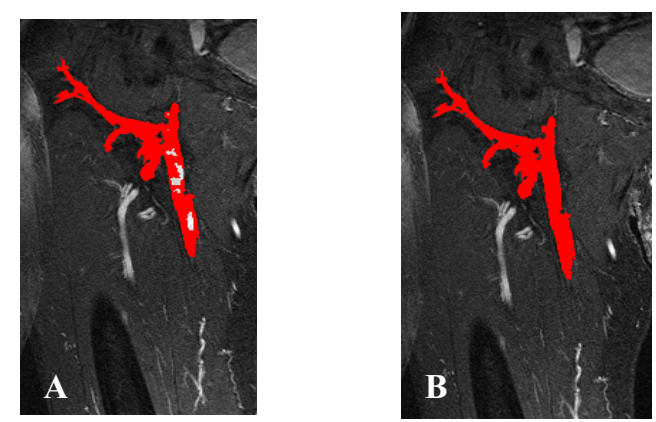

Fig. 8. Isocontour segmentation of the MRA image in Figure 7 by using the $\chi_{\mathrm{a}}$ formula (A) and the $\beta_{\mathrm{a}}$ formula $(\mathbf{B})$. In Fig. $\mathbf{B}$, the extracted venous structures show no discontinuities or holes

This example indicates the validity of proposed new formula for images containing structures and objects with high grey levels, like blood vessels. The $\beta$-connectedness does not compel the user to choose a particular seed point to obtain a segmentation 
result without holes or discontinuities. In Fig. 7, the selected seed point shows a low grey level but the vessel has been extracted with high precision.

In the next picture (Fig.9), it is possible to see a comparison between the result of the segmentation technique described in Section 4 (Player function) and the result of the classical Isocontour algorithm proposed in [2]: both results have been obtained by using the $\beta_{\mathrm{a}}$-connectedness over an MRA image.

As is easy to notice the Player function enables one to understand the temporal evolution of the segmentation process by following the sequence of colours used (i.e., green, red, blue and yellow). Moreover, this function results in sharpen edges of the structure of interest.
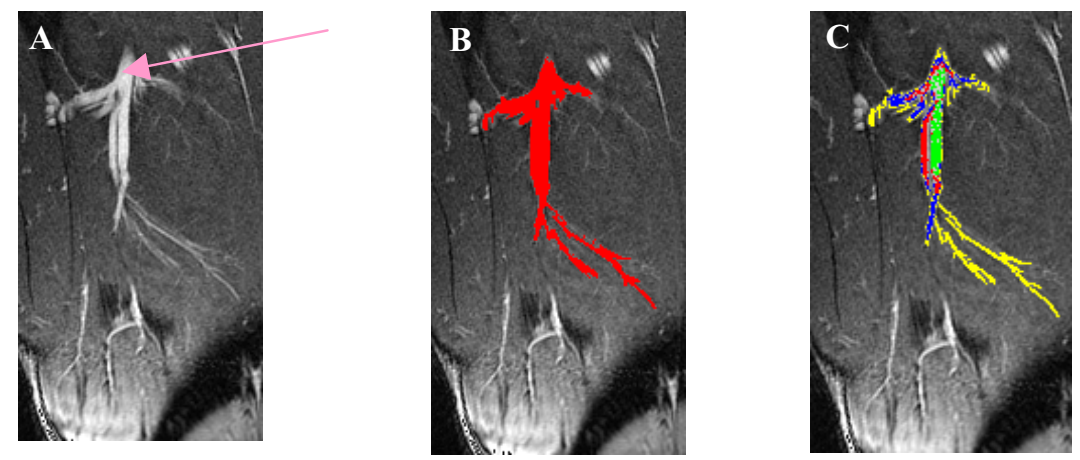

Fig. 9. Original image and selected seed point (arrow) (A). Segmentation result yielded by the thresholding operation after the $\beta_{\mathrm{a}}$ field calculation (B). Segmentation result obtained by using the hierarchical tree $(\mathbf{C})$ : the Player function allows the vessel walls to be extracted with the highest precision

In Fig. 10, a 3D visualization is presented (surface rendering technique) to compare a classical threshold applied to the formula (A) with the Player function mechanism (B). The new concept of connectivity used for the tree growth allows one to extract the same vessel with a better accuracy.
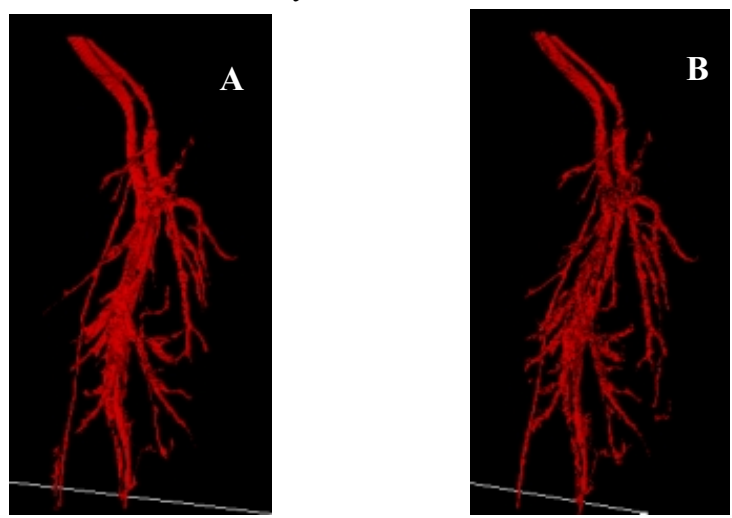

Fig. 10. 3D visualization using Isocontour (A) and the Player function (B) 


\section{Conclusions}

The proposed algorithm, based on the -connectedness computation, is able to better analyze bright structures, thus showing a greater effectiveness in segmenting blood vessels in CE-MRA images. Moreover, the connectivity computation, as proposed in a previous work [2], has been exploited here to realize an original organization of the extracted data into a hierarchical tree that allows one to segment the structure of interest with a very high precision, even under low contrast conditions. The results show that this algorithm can provide a promising starting point to solve the vessel/artery separation problem.

Finally, the exploitation of the pixel hierarchical growth and of the father-son relationships is an innovative strategy that is presented to the scientific community for the first time, and makes it possible to improve segmentation results in critical cases.

\section{References}

1. Rosenfeld, A.: The fuzzy geometry of image subset. Pattern Recognition Lett. (1984) 2, 311-317

2. Dellepiane, S., Fontana, F., and Vernazza, G.: Nonlinear image labeling for multivalued segmentation. IEEE Transactions on Image Processing, Vol. 5, No. 3, March 1996

3. Dellepiane, S., and Fontana, F. Extraction of intensity connectedness for image processing. Pattern Recognition Letters (1995) 16, 313-324

4. Udupa, J.K., and Samarasekeba, S.: Fuzzy connectedness and object definition: theory, algorithms and applications in image segmentation. Graphical Models and Image Processing, Vol. 58, No.3, May, pp.246-261, 1996

5. Lei, T., Udupa, J. K., Saha, P. K., and Odhner, D.: Artery-vein separation via MRA- an image processing approach. IEEE Transactions on Medical Imaging, Vol.20, No.8, August 2001

6. Yim, P.J., Choyke, P.L., and Summers, R.M.: Gray-scale skeletonization of small vessels in Magnetic Resonance Angiography. IEEE Transactions on Medical Imaging, Vol. 19, No. 6, June 2000

7. Stefancik, R.M., Sonka, and M.: Highly Automated segmentation of arterial and venous trees from three-dimensional magnetic resonance angiography (MRA). The International Journal of Cardiovascular Imaging 17: 37-47, 2001 